\title{
Workshop Gesundheitsgefährdung durch langes Sitzen am Arbeitsplatz - Teil I wissenschaftliche Perspektiven
}

\section{Workshop on Health Risks of Sedentary Behavior at Work - Part I} Scientific Perspectives

\section{(c) (i)}

\section{Autoren}

Ute Latza', Jens Bucksch²,3, Birgit Wallmann-Sperlich4

Institute

1 Fachbereich Arbeit und Gesundheit, Bundesanstalt für Arbeitsschutz und Arbeitsmedizin, Berlin

2 Fakultät III - Studiengang Prävention und Gesundheitsförderung, Padagogische Hochschule Heidelberg, Heidelberg

3 WHO Collaborating Center for Child and Adolescent Health Promotion, Universität Bielefeld Fakultät fur Gesundheitswissenschaften, Bielefeld

4 Institut für Sportwissenschaft, Julius-Maximilians-Universität Würzburg, Würzburg

\section{Schlüsselwörter}

Sitzen, sedentäres Verhalten, Arbeitswelt, Intervention, Prävention, Betriebliche Gesundheitsförderung

\section{Key words}

Sitting, sedentary behavior, work, intervention, prevention, workplace health promotion

\section{Bibliografie}

DOI https://doi.org/10.1055/a-1173-9001

Gesundheitswesen 2020; 82: 623-631

(c) Georg Thieme Verlag KG Stuttgart · New York

ISSN 0941-3790

\section{Korrespondenzadresse}

Prof. Ute Latza

Bundesanstalt für Arbeitsschutz und Arbeitsmedizin

Fachbereich Arbeit und Gesundheit

Nöldnerstraße 40-42

10317 Berlin

latza.ute@baua.bund.de

\section{ABSTRACT}

Ziel Die Gesundheitsgefährdung durch sedentäres Verhalten am Arbeitsplatz ist ein wichtiges Problem unserer Zeit. Seine Lösung verlangt nach interdisziplinärer Zusammenarbeit von Expertinnen und Experten aus Wissenschaft und betrieblicher Praxis.
Methodik Hierzu wurde im Februar 2019 in Berlin ein Workshop mit Fachleuten aus verschiedenen Bereichen durchgeführt, der die derzeitigen wissenschaftlichen Erkenntnisse der Gesundheitsgefährdung durch lange, wenig unterbrochene Sitzzeiten am Arbeitsplatz thematisiert und ausgewählte betriebliche Lösungsansätze aufzeigt. Dieser Workshop wird hier in 2 Teilen dokumentiert. Genderrelevante Aspekte werden, wenn möglich, hervorgehoben.

Ergebnisse Im vorliegenden Teil I werden die Beiträge zu aktuellen wissenschaftlichen Erkenntnissen bezogen auf die Gesundheitsrisiken durch sedentäre Lebens- und Arbeitsweisen sowie Prävalenz und Determinanten für sedentäres Verhalten in der Arbeitswelt referiert. Die darauf aufbauenden Beiträge zu theoriegeleiteten Interventionsstrategien und zur Evidenz für erfolgreiche Interventionsstrategien aus systematischen Reviews zeigen mögliche Lösungswege auf.

Schlussfolgerung und Ausblick Trotz vieler Studien und Hinweise, die für eine stärkere Berücksichtigung des sedentären Verhaltens im betrieblichen Kontext sprechen, gibt es Wissenslücken v.a. bezogen auf Ätiologie, Möglichkeiten der Kompensation, Determinanten und Interventionsstrategien. Auch sind Unterschiede zwischen Männern und Frauen in diesem Zusammenhang unzureichend dokumentiert. Im Teil II werden ausgewählte betriebliche Lösungsansätze und die Ergebnisse der Workshop-Diskussion vorgestellt.

\section{ABSTRACT}

Aim Health risks of sedentary behaviour at work are a matter of concern. Solutions are needed based on interdisciplinary collaborations of research experts and practitioners in the field of occupational safety and health.

Methods In February 2019, at a workshop in Berlin, experts from different fields discussed the health risks of sedentary behaviour at work and solutions for the workplace based on scientific evidence. The workshop is documented here in 2 parts. Gender relevant aspects are distinguished if possible. Results Part I describes the workshop contributions on current scientific findings regarding health risks of sedentary behavior in leisure time and at work as well as prevalence and determinants of sedentary work. The following contribution 
presents suggestions for the development of theory-based intervention strategies. Furthermore, available evidence from systematic reviews for intervention strategies to reduce sedentary time at work is shown.

Conclusion and outlook In spite of many studies and evidence in favour of taking into consideration sedentary behav- ior at work, there are still research gaps predominantly regarding etiology, possibilities of compensation, determinants, and intervention strategies. In this context, documentation of differences between men and women is insufficient. Part II presents selected workplace solutions and the results of the discussions in the workshop.

\section{Einleitung}

Die Gesundheit der Erwerbsbevölkerung stellt eine wichtige Ressource für die Arbeitswelt und den Wirtschaftsstandort Deutschland dar. Mehr als die Hälfte der Bevölkerung ist erwerbstätig und kann von gesundheitsförderlichen Arbeitsplätzen profitieren. Allerdings verbringen immer mehr Menschen den größten Teil ihres Arbeitstages und ihre Freizeit sedentär. Damit ist langes, wenig unterbrochenes Sitzverhalten gemeint. Der Begriff sedentäres Verhalten leitet sich aus englisch „sedentary behaviour“ ab. Sedentäres Verhalten führt zu Risiken u.a. für das Herz-Kreislauf-System und den Stoffwechsel und einem Anstieg an chronischen nichtübertragbaren Erkrankungen (wie Diabetes Typ 2). Nach einer aktuellen Prognose des Robert Koch-Instituts werden im Jahr 2040 über 10 Mio. Menschen in Deutschland von einer Typ-2-DiabetesErkrankung im Vergleich zu knapp 7 Mio. im Jahr 2015 betroffen sein [1]. Sedentäres Verhalten im betrieblichen Kontext zu reduzieren, stellt dabei eine wichtige Möglichkeit der Prävention von Stoffwechselerkrankungen dar.

Da sich sowohl Arbeitsmarkt, Gesundheitsrisiken sowie Sitz- und Bewegungsverhalten für Männer und Frauen stark unterscheiden, sind bei Themen der Prävention und Gesundheitsförderung wie die Reduzierung des sedentären Verhaltens, die Verschränkung von biologischem (Sex) und sozialem Geschlecht (Gender) zu betrachten (Sex/Gender-Perspektive) [2, 3]. Im Rahmen der Förderrichtlinie des Bundesministeriums für Bildung und Forschung (BMBF) „Gesund - ein Leben lang“, Fördermaßnahme „Gendergesundheit“ fand am 13. Februar 2019 der Workshop „Gesundheitsgefährdung durch langes Sitzen am Arbeitsplatz - wissenschaftliche Perspektiven und betriebliche Lösungsansätze“ in Berlin statt. Zu dem Workshop hatte die Bundesanstalt für Arbeitsschutz und Arbeitsmedizin (BAuA) als Projektnehmerin in Kooperation mit dem Institut für Public Health der Charité Universitätsmedizin Akteure aus dem betrieblichen Arbeits- und Gesundheitsschutz sowie aus Public Health als Referierende bzw. Teilnehmende eingeladen (z. B. Bundesministerium für Arbeit und Soziales, Beauftragte für Betriebliches Gesundheitsmanagement und Betriebsärztinnen und -ärzte verschiedener deutscher Firmen, Wissenschaftler und Wissenschaftlerinnen aus unterschiedlichen Universitäten bzw. Forschungseinrichtungen inkl. der BAuA sowie Anbieter von Gesundheitsdienstleitungen und ergonomischer Lösungen für Büroarbeitsplätze). Die Beiträge und Diskussionen werden hier in 2 Artikeln zusammengefasst.

Ziel des Workshops war es, weiterführende Impulse für das Thema der körperlichen Unterforderung durch sedentäre Arbeit in die Bereiche der betrieblichen Gesundheitsförderung und des Arbeitsschutzes zu setzen. Aktuell sind physische Überforderung bezogen auf das Muskel-Skelett-System und psychische Über- und
Unterforderung bezogen auf die psychische Gesundheit auf der Agenda der Akteure des Arbeitsschutzes und der betrieblichen Prävention und Gesundheitsförderung. Sedentäre Arbeit wird dabei häufig als traditionelles Forschungs- und Handlungsfeld missverstanden, dem nur mit bekannten Methoden der Ergonomie und Bewegungsförderung begegnet werden sollte. Die Dimension der metabolischen Unterforderung mit den neuen Möglichkeiten ihrer Prävention und ihren Konsequenzen für die Gestaltung von Arbeit müssen verstanden und transdisziplinär weiterentwickelt werden.

Im vorliegenden Teil I wird zunächst in die wissenschaftliche Relevanz des Themas eingeführt. Hierfür werden im ersten referierten Workshop-Beitrag der Arbeitsepidemiologin Ute Latza Gesundheitsrisiken durch sedentäre Lebens- und Arbeitsweisen beschrieben. Im folgenden Beitrag der Sportwissenschaftlerin Birgit Sperlich werden Häufigkeit und Determinanten für lange Sitzzeiten aufgezeigt. Darauf aufbauend wird im dritten Beitrag des Gesundheitswissenschaftlers Jens Bucksch vorgestellt, wie Interventionsstrategien theoriegeleitet entwickelt werden könnten und für welche Strategien Evidenz vorliegt. Die Beiträge beziehen sich narrativ auf den aktuellen Stand der Forschung. Da wo vorhanden greifen sie auf Wissen aus systematischen Übersichtsarbeiten zurück und ergänzen diese durch Primärstudien zu spezifischen Teilaspekten. Vor dem Hintergrund der Unterschiede bezogen auf Sex/Gender versuchen die Beiträge entsprechend dazu vorliegende spezifische Befunde zu berücksichtigen. Eine Auseinandersetzung mit Sex/Genderbias zum Thema Sitzen erfolgt an anderer Stelle [4].

Im Teil II [5] werden ausgewählte betriebliche Lösungsansätze und exemplarische Beispiele vorgestellt.

\section{Gesundheitliche Risiken durch sedentäres Verhalten}

Sedentäres Verhalten wird in einer Konsensus-Definition als geringfügig energetisch beanspruchende Lebens- und Arbeitsweise der Wachzeit ( $\leq 1,5$ metabolische Äquivalente [MET], z. B. in aufrecht oder zurückgelehnter Sitzposition) beschrieben [6, 7]. Sedentäre Verhaltensweisen können in allen Lebensbereichen vorkommen, v.a. Arbeit, Transport und Freizeit.

Sedentäres Verhalten muss von Bewegungsmangel bzw. körperlicher Inaktivität abgegrenzt werden. Entsprechend den aktuellen nationalen Bewegungs-Empfehlungen liegt Bewegungsmangel dann vor, wenn die Minimalempfehlungen für körperliche Aktivität nicht erreicht werden [8]. Wie viele nationale Empfehlungen basieren diese auf denen der Weltgesundheitsorganisation [9], die hinsichtlich der aeroben körperlichen Aktivität $\geq 150$ min/Woche Bewegung mit moderater Intensität (3-6 MET) oder $\geq 75 \mathrm{~min} /$ Woche mit hoher Intensität (d.h. anstrengender körperlicher Ak- 
tivität, $\geq 6 \mathrm{MET}$ ) sowie jegliche Kombination beider für Erwachsene im Alter von 18 bis 64 Jahren empfehlen. Die körperliche Aktivität sollte dabei in mindestens 10-minütigen Umfängen stattfinden $[8,9]$. Energetisch zwischen sedentärem Verhalten und moderat-intensiver körperlicher Aktivität liegen die leicht intensiven körperlichen Aktivitäten (z. B. langsames Gehen, 1,5-3 MET), die für die Unterbrechung des sedentären Verhaltens eine bedeutende Rolle spielen können.

Beide Verhaltensweisen - Bewegungsmangel und sedentäres Verhalten - können koexistieren. So kann bspw. eine Managerin 70 \% ihrer 10-stündigen Arbeitszeit am klassischen Büroschreibtisch und abends noch einmal 3 Stunden in sozialen Medien, also 10 Stunden pro Tag, sitzend verbringen und mit einer Stunde Fitnessstudio pro Tag körperlich dennoch hoch aktiv sein und gleichzeitig eine sedentäre Lebensweise aufweisen. Demgegenüber weist eine Reinigungskraft aufgrund ihrer körperlich aktiven Arbeitsweise keinen sedentären Lebensstil während der Arbeit auf, kann allerdings an Bewegungsmangel leiden, wenn sie in Ihrer Freizeit nicht der empfohlenen körperlichen Aktivität nachgeht.

\section{Gesundheitsrisiken sedentärer Lebens- und Arbeitsweisen}

Generell steigert eine ausgeprägte sedentäre Lebens- und Arbeitsweise das Risiko für die gesellschaftlich dominierenden chronischen Erkrankungen sowie eine erhöhte frühzeitige Sterblichkeit. DosisWirkungsbeziehungen sind v.a. für den Zusammenhang zur Gesamtmortalität [10] sowie für Herz-Kreislauf Mortalität und Inzidenz von Diabetes Typ 2 beschrieben [exempl. 11].

Aktuellen Studien zufolge wird sedentäres Verhalten als Risikofaktor beschrieben, der unabhängig vom Erreichen der Mindestaktivitätsempfehlungen wirkt [12]. Ein zentraler Beleg hierfür ist eine
Metaregression mit Daten aus prospektiven Studien mit mehr als 1 Mio. Personen. Ekelund et al. [13] zeigen für sedentäre Lebensund Arbeitsweisen (d.h. nicht differenziert dafür, wo gesessen wurde) einen erwartungskonformen Anstieg der Mortalität mit Abnahme der körperlichen Aktivität (hier gemessen als Quartile pro Woche basierend auf Selbstangaben) ( $\mathbf{A b b}$. 1). Zusätzlich berücksichtigten die Autoren die Dauer des Sitzens (angegeben als Stunden/Tag basierend auf Fragebogenangaben). Dabei beobachteten sie einen weiteren Gradienten: Auch bei den Personen, die entsprechend der WHO-Empfehlung [9] körperlich aktiv waren, zeigte sich ein Anstieg der Sterblichkeit mit zunehmender Sitzdauer. Dabei ist zu bedenken, dass weniger als die Hälfte der Deutschen in diesem Ausmaß aktiv sind [14]. Nur bei Personen, die angegeben hatten, stark körperlich aktiv zu sein (ungefähr 3-fach der WHO-Empfehlung [9], also ungefähr jeden Tag ca. eine Stunde Fitnessstudio), wurde kein Anstieg der Mortalität mit zunehmender Sitzdauer beobachtet. Nur sehr wenige Personen erreichen dieses Ziel tatsächlich.

Modelle zu biologischen Wirkmechanismen, die sedentäres Verhalten mit veränderten kardiometabolischen Prozessen der Unterforderung verbinden, basieren v. a. auf laborexperimentellen Tierund wenigen Humanversuchen $[15,16]$. Eine Metaanalyse humaner Laborstudien zeigt, dass das Unterbrechen langer Sitzzeiten durch Phasen im Stehen oder Gehen sich positiv auf metabolische Blutwerte (Glukose, Insulin, Insulinsensitivität) auswirken kann [17]. Eine Querschnittstudie gibt erste Hinweise auf mögliche positive Effekte bezogen auf die Entwicklung eines Typ-2-Diabetes [18].

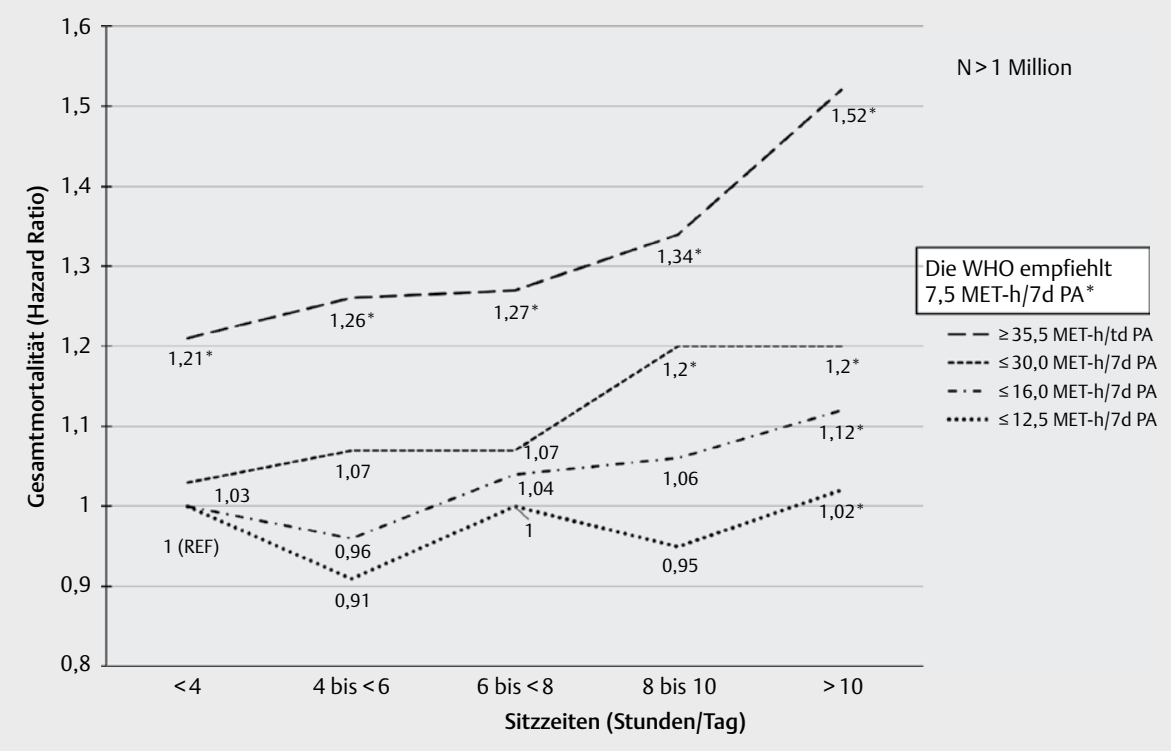

- Abb. 1 Metaanalyse zum gemeinsamen Zusammenhängen von Sitzzeit und körperlicher Aktivität (kA) mit der Gesamtsterblichkeit (Daten aus [13]; mit freundlicher Genehmigung von Hidde van der Ploeg 2018). Legende: Metabolische Äquivalente (MET) angegeben in Stunden pro Woche (h/7d). Tägliches Sitzen mit einer Dauer von 6-8 Stunden ist ein Risiko für vorzeitige Mortalität. 


\section{Bedeutung sedentärer Arbeitsweisen für die Arbeitswelt}

Aufbauend auf der Evidenz zu Gesundheitsrisiken durch generell sedentäre Leben- und Arbeitsweisen gibt es zunehmend Hinweise, dass dieser Zusammenhang auch für berufliches Sitzen besteht. Der aktuellste systematische Review zu dieser Frage [19] zeigt Hinweise aus prospektiven Studien für den Zusammenhang von beruflichen Sitzzeiten mit erhöhter Sterblichkeit und Diabetes Typ 2 auf. Insgesamt besteht aufgrund von Qualitätsmängeln jedoch weiterer Forschungsbedarf. Aktuell wird ein Update des systematischen Reviews zur Ätiologie von kardiometabolischen Erkrankungen bzw. Veränderungen unter Berücksichtigung von Unterschieden zwischen den Geschlechtern im Rahmen eines BMBF-geförderten Projekts ${ }^{1}$ durchgeführt. Das Projekt ${ }^{2}$ ist bei Prospero registriert ${ }^{3}$ und soll 2019 abgeschlossen werden. Wenn gezeigt werden kann, dass körperliche Unterforderung durch lange Sitzzeiten am Arbeitsplatz ein Risikofaktor ist, hat dies Konsequenzen für die Arbeitswelt.

Aus Public Health Perspektive kann Gesundheitsförderung im Setting Arbeitswelt dazu beitragen, soziale Ungleichheit abzubauen und auch sozial benachteiligte Bevölkerungsgruppen durch Empowerment und Stärkung von Ressourcen zu fördern [20]. Das Präventionsgesetz aus dem Jahr 2015 [21] soll dazu beitragen, in Zusammenarbeit mit Sozialversicherungsträgern und Arbeitsschutz die Betriebliche Gesundheitsförderung zu verbessern. Zu den klassischen verhaltenspräventiven, individuellen Maßnahmen der Betrieblichen Gesundheitsförderung gehören Angebote im Bereich der Bewegungsförderung. Während die Gesundheitsförderung im betrieblichen Setting für gesetzliche Kranken- und Unfallversicherungsträger verpflichtend ist, ist diese freiwillig für die betrieblichen Partner.

Der betriebliche Arbeits- und Gesundheitsschutz ist komplex gesetzlich geregelt. Im Betrieb trägt der Arbeitgeber die Verantwortung für Sicherheit und Gesundheit der Beschäftigten. Sicherheitsfachkräfte und die Betriebsmedizin können ihn bei dieser verpflichtenden Aufgabe beraten. Kollektive Beschäftigtenvertretungen (Betriebs-/Personalrat) vermitteln häufig die Partizipation der Beschäftigten. Arbeitsschutzziele sind die gesundheitsförderliche und menschengerechte Gestaltung der Arbeit. Wie aus der Perspektive von Public Health bzw. der Gesundheitsförderung liegt auch bei den basierend auf einer Gefährdungsbeurteilung abgeleiteten Arbeitsschutzmaßnahmen die Priorität auf primärpräventiven Maßnahmen (d.h. technische Änderung der Arbeitsumgebung und geänderter Arbeitsorganisation) (siehe auch [22] § 4). Diese können durch Maßnahmen der individuumsbezogenen Verhaltensprävention und der betrieblichen Gesundheitsförderung unterstützt werden.

$1 \quad$ BMBF. Bundesministerium für Bildung und Forschung. Präventions- und Ernährungsforschung, Gendergesundheit, Einzelprojekt [Online: www. gesundheitsforschung-bmbf.de/de/sitting-work-dauersitzen-korperliche-inaktivitat-am-arbeitsplatz-und-die-wirkung-auf-6712.php]

2 BAuA. Bundesanstalt für Arbeitsschutz und Arbeitsmedizin. Forschungsprojekte [Online: www.baua.de/DE/Aufgaben/Forschung/Forschungsprojekte/f2399]

3 PROSPERO International prospective register of systematic reviews, National Institute for Health Research, CRD42018079219 [Online: https:// www.crd.york.ac.uk/prospero/display_record.php?RecordID = 79219]
Bisher wurde Sitzen am Arbeitsplatz vorrangig im Sinne einer physischen Überforderung durch Zwangshaltungen betrachtet. Dabei orientiert sich die Gefährdungsbeurteilung einer Tätigkeit bzw. eines Arbeitsplatzes bisher nur an Obergrenzen der Belastung, d. h. den Folgen körperlicher Überforderung meist bezogen auf Beschwerden des Bewegungsapparats. Bisher wurde keine Untergrenze für den Energieverbrauch definiert und es existieren nur indirekte Gestaltungsempfehlungen (über das Arbeitszeitgesetz).

Neue Denkansätze zur Vermeidung von körperlicher Über - als auch Unterforderung werden in der Diskussion in Teil II [5] angesprochen. Eine vollständige Substitution von Sitzen durch Stehen ist allerdings keine Option, denn die Prävention der Belastungen und Erkrankungen (z. B. venöse Erkrankungen der Beinvenen) durch Stehen am Arbeitsplatz ist ein langjähriges Ziel des präventiven Arbeits- und Gesundheitsschutzes [23].

\section{Prävalenz von Sitzzeiten und ihre Determinanten}

Seit den 60er Jahren hat die Zeit in sedentärer Verhaltensweise bei gleichzeitigem Rückgang von körperlicher Aktivität insgesamt als auch insbesondere während der Arbeit beträchtlich zugenommen (> $40 \%$ in einigen Ländern) [24]. Dies spiegelt unter anderem die stetigen Veränderungen der Arbeitswelt mit vermehrter Computerisierung, Technisierung und Modernisierung von Arbeitsaufgaben wider, die sich in gravierenden Veränderungen in dem körperlichen Aktivitätsprofil der arbeitenden Bevölkerung niederschlägt.

Die Sitzzeiten der Erwerbsbevölkerung während der Arbeitszeit variieren je nach Berufsgruppe stark $[25,26]$ und liegen einer aktuellen Metaanalyse zufolge über alle Berufsgruppen hinweg bei einem Sitzanteil während der Arbeitszeit von ca. 60 \% bzw. für die gesamte Wachzeit bei 59\% [26]. Erwerbstätige, die einen Büroarbeitsplatz einnehmen, weisen den höchsten Sitzanteil während der Arbeitszeit (73\%) bzw. der gesamten Wachzeit (66\%) auf [26], so dass insgesamt Sitzzeiten von über 11 Stunden an Werktagen festzustellen sind [27]. Gleichzeitig wiesen Erwerbstätige an Büroarbeitsplätzen den geringsten Anteil an leichter körperlicher Aktivität während der Arbeitszeit (13\%) und während der gesamten Wachzeit (23\%) auf, aber die höchste körperliche Aktivität in mindestens moderater Intensität während der Wachzeit (45 Min. pro Tag, davon 11 Min. während der Arbeitszeit) [26]. Die sedentäre Arbeits- und Lebensweise von Erwerbstätigen an Büroarbeitsplätzen zeigt, dass lange Sitzzeiten während der Arbeit nicht durch kürzere Sitzzeiten und mehr leichte körperliche Aktivität außerhalb der Arbeit kompensiert werden.

Hinsichtlich der Analyse von möglichen Einflussfaktoren auf das sitzende Verhalten am Arbeitsplatz wird einem sozial-ökologischen Ansatz gefolgt $[28,29]$. Dieser unterscheidet zwischen Einflussfaktoren der personenbezogenen Ebene (z. B. Soziodemografie, Biologie, sozial-kognitive Eigenschaften wie Einstellungen gegenüber Sitzen oder Gesundheitswissen bzgl. Sitzen am Arbeitsplatz), der soziokulturellen Ebene (z. B. Arbeitsplatznormen, Arbeitskultur, kollegiale und Führungsunterstützung), der Arbeitsplatzumgebung (z. B. Büroausstattung, Meetingräume, Treppenhäuser) und der politischen Rahmenbedingungen (z. B. Arbeitsrechtliche Vorgaben, Pausenregelungen) (\ Abb. 2). 


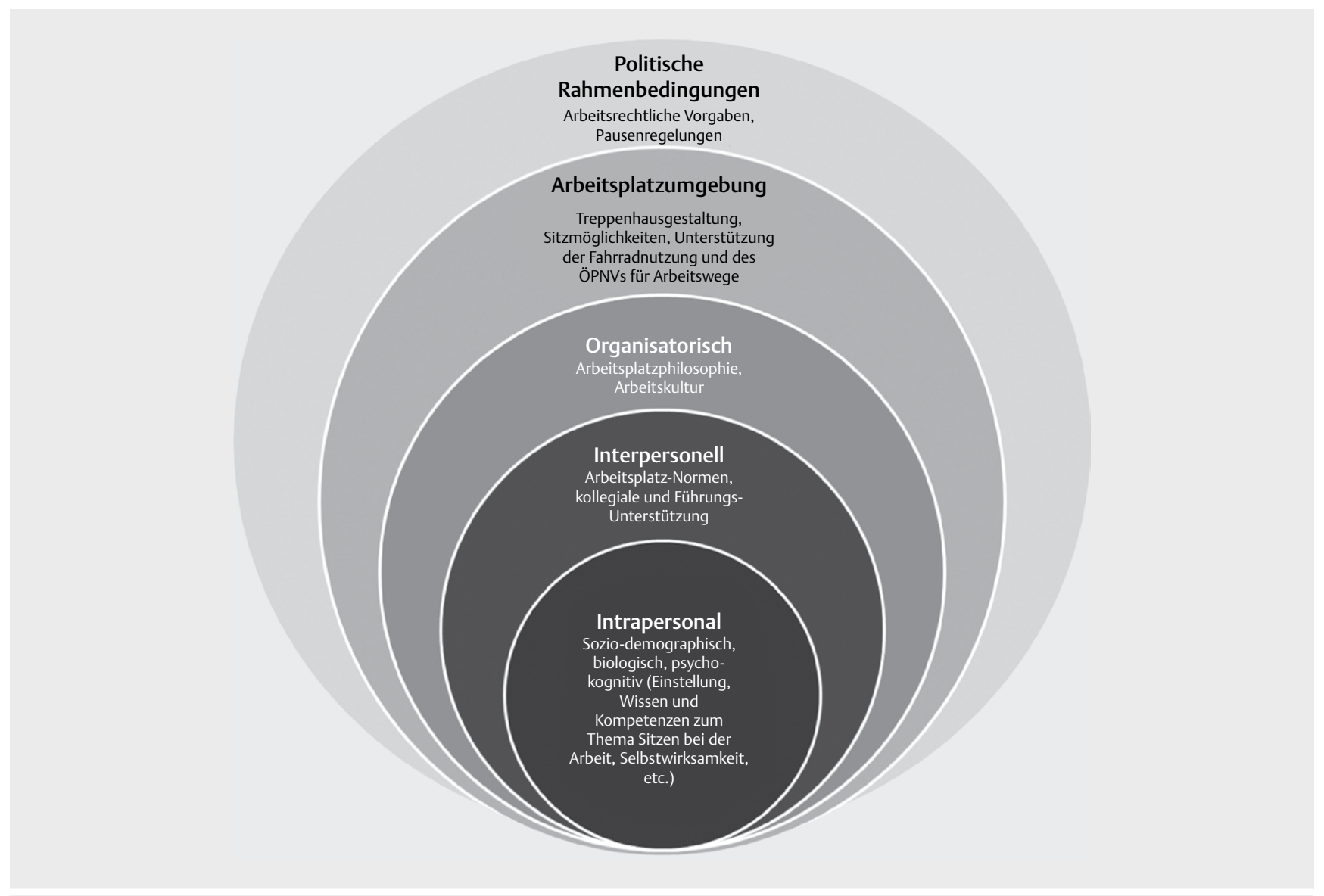

- Abb. 2 Determinanten von sedentärem Lebensstil im Arbeitskontext (Daten aus [28]).

Eindeutig ist die Studienlage hinsichtlich der sozio-demographischen Einflussfaktoren. Personen mit höherem Bildungsniveau und Einkommen weisen höhere arbeitsplatzbezogene Sitzzeiten auf $[30,31]$ sowie Beschäftigte in sogenannten „White-Collar vs. BlueCollar"-Berufen bzw. Berufstätige in Vollzeit- vs. Teilzeitstellen $[30,31]$. Vereinzelte Hinweise gibt es hinsichtlich biologischer Determinanten. Der Body Mass Index scheint mit den Sitzzeiten am Arbeitsplatz positiv zu korrelieren [32]. Einzelne Studien deuten zudem darauf hin, dass je jünger die Berufstätigen, desto höher sind auch die Sitzzeiten am Arbeitsplatz [32]. In einer geschlechtsspezifischen Analyse der arbeitsbezogenen Sitzzeiten in Deutschland ergab sich dies allerdings nur für die Frauen [33]. Insgesamt gibt es wenige Untersuchungen, die geschlechtsspezifisch die Determinanten von Sitzzeiten am Arbeitsplatz untersuchen.

Studien zeigen auch, dass die Einstellungen zum Sitzen am Arbeitsplatz mit den tatsächlichen Sitzzeiten in Zusammenhang stehen. De Cocker et al. [32] dokumentieren, dass eine hohe wahrgenommene Kontrolle über die eigenen Arbeitssitzzeiten mit geringeren Arbeitssitzzeiten korreliert. Das Wahrnehmen von Vorteilen von Sitzen am Arbeitsplatz sowie die hohe persönliche Intention, Sitzzeiten am Arbeitsplatz zu reduzieren, korrelieren positiv mit Arbeitsplatzsitzzeiten in einer weiteren Einzelstudie [31].

Determinanten auf Ebene der Arbeitsumgebung lassen sich insbesondere aus Interventionsstudien ableiten. Als wichtige Einfluss- faktoren für die Sitzzeitreduzierung zeigen sich aktive Arbeitsplätze wie z. B. elektrisch höhenverstellbare Schreibtische, Software, die regelmäßig ans Aufstehen erinnert oder Pedalsysteme unter dem Schreibtisch, die zu einer Reduzierung der Sitzzeiten am Arbeitsplatz führte [31,34]. Die Bereitstellung eines höhenverstellbaren Schreibtischs bedeutet jedoch nicht notwendigerweise, dass dieser auch tatsächlich genutzt werden [35].

Hinsichtlich soziokultureller Determinanten (z. B. einer Arbeitskultur mit Stehmeetings [36], soziale Normen am Arbeitsplatz) oder Einflussfaktoren auf der arbeitspolitischen Ebene existieren bisher unseres Wissens nach noch keine Studien.

\section{Konzeptionelle Überlegungen und vorliegende Studien zu Interventionsstrategien}

Generell fehlt bisher hochwertige Evidenz für die Wirksamkeit von betrieblichen Interventionen bezogen auf die Reduktion des sedentären Verhaltens und auf positive gesundheitsförderliche Effekte [34, 36]. Obwohl Multikomponenten-Interventionsstrategien beschrieben sind, die auf verschiedenen Ebenen der Verhältnisund Verhaltensprävention ansetzen sind dabei Veränderung der Arbeits- und Organisationskultur nur selten beschrieben [37]. Eine ganzheitliche Verknüpfung von Maßnahmen zur Reduktion von sedentärem Verhalten am Arbeitsplatz mit Maßnahmen der Präven- 


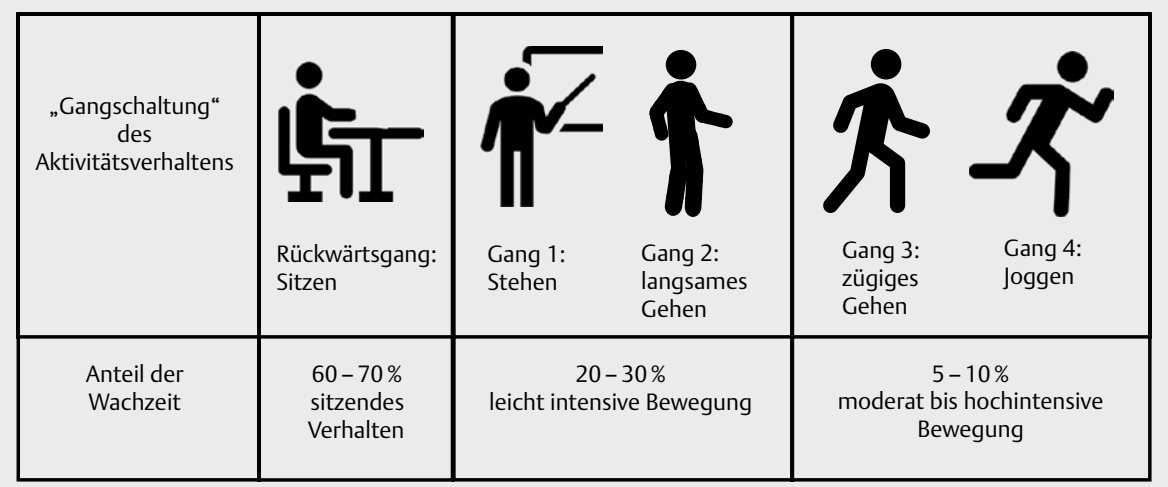

- Abb. 3 Gangschaltung des Aktivitätsverhaltens. Nach dem Konzept von Buckley JP, Hedge A, Yates T et al. [50] wird es - in Analogie zum Autofahren - kaum gelingen, vom Rückwärtsgang (= sedentäres Verhalten) in den 3. (= zügiges Gehen) oder 4. Gang (= Joggen) zu schalten.

tion psychosozialer Risiken und Förderung psychosozialer Ressourcen steht noch aus.

International wird insbesondere im betrieblichen Kontext von Büroangestellten mit verschiedenen Interventionsansätzen auf den sedentären Arbeitsalltag reagiert. Aus Übersichtsarbeiten kristallisiert sich heraus, dass bewegungsassoziierte Interventionsansätze (z. B. Laufgruppe, erleichterter Zugang zu Fitnessstudios) nur unzureichende Wirkungen auf das sedentäre Verhalten am Arbeitsplatz ausüben. Der Interventionserfolg ist höher, je spezifischer die Maßnahmen darauf angelegt sind, lange Sitzzeiten regelmäßig zu unterbrechen oder Arbeit im Stehen bzw. in Bewegung durchzuführen [38-40]. Der Schlüssel zum Erfolg liegt somit in einer Verschiebung der sedentär verbrachten Zeit zu den sogenannten alltäglichen Basisaktivitäten [40]. Es lässt sich somit festhalten, dass sedentäres Verhalten reduzieren und Bewegung zu fördern, zwei sich ergänzende Zugänge mit eigener Fokussierung und Logik sind. Es wird somit eine Doppelstrategie benötigt. Die beiden Zugänge dürfen nicht gegeneinander ausgespielt werden [40,41]. Zusammenfassend lässt sich das in nachfolgender Abbildung über die Gangschaltung des Aktivitätsverhaltens veranschaulichen. In Analogie zum Autofahren wird es kaum gelingen vom Rückwärtsgang ( = sedentäres Verhalten) in den 3. (=zügiges Gehen) oder 4. Gang ( = Joggen) zu schalten ( $\triangleright$ Abb. 3 ).

Diese sich aus Wirksamkeitsüberlegungen abgeleiteten Abgrenzungen lassen sich auch verhaltenswissenschaftlich begründen ( $\triangleright$ Tab. 1). Sedentäres Verhalten ist durch einen niedrigen Anstrengungsgrad und von langer Dauer im Tagesverlauf geprägt und unterliegt einem geringen Grad der bewussten Steuerung. Es handelt sich um ein Verhalten mit hoher Gewohnheitsstärke. Sport und gesundheitsförderliche Aktivität hingegen sind im Verhältnis von kurzer Dauer und treten nur gelegentlich im Tagesverlauf auf. Der Grad der Anstrengung und der bewussten Steuerung ist hoch. Dies impliziert weitergehend, dass klassische sozial-kognitive Theorien sedentäres Verhalten nicht ausreichend erklären. Theoretisch ist es lohnenswert, gewohnheitsbildende Elemente und damit verbunden die Bedeutung von Mikroumwelten in die Erklärung von sedentären Verhalten zu fokussieren. Interventionen werden nur dann
- Tab. 1 Sport und sedentäres Verhalten aus verhaltenswissenschaftlicher Sicht (Daten aus [41]).

\begin{tabular}{|l|l|l|}
\hline Qualität & $\begin{array}{l}\text { Strukturierte } \\
\text { Bewegungseinhei- } \\
\text { ten/Sport }\end{array}$ & $\begin{array}{l}\text { Sedentäres } \\
\text { Verhalten }\end{array}$ \\
\hline Häufigkeit im Tagesverlauf & - & ++ \\
\hline Dauer & - & ++ \\
\hline Anstrengungsgrad & $+1++$ & - \\
\hline $\begin{array}{l}\text { Grad der bewussten } \\
\text { Steuerung }\end{array}$ & $+/++$ & $-/-$ \\
\hline $\begin{array}{l}|l| \\
\text {-- unbewusst/Gewohnheit. - gering bzw. niedrig bzw. kurz. + } \\
\text { moderat. + + hoch bzw. lang }\end{array}$ \\
\hline
\end{tabular}

replizierbar und nachhaltig sein, wenn sie stärker als bisher an den theoretischen Vorüberlegungen ansetzen [40-42].

Die derzeitige Evidenz zu den spezifischen Interventionen zur Reduzierung des sedentären Verhaltens im Allgemeinen und bei schreibtischbezogener Arbeitszeit zeigt, dass Maßnahmen dann erfolgreich sind, wenn sie sowohl die Umwelt verändern (z. B. höhenverstellbare Tische) als auch verschiedene weitere Interventionsebenen parallel bedienen, wie Veränderungen der Arbeitsorganisation, Information und Beratung $[43,44]$. Sie also entsprechend der sozial-ökologischen Theorie angelegt sind (s.o. „Prävalenz von Sitzzeiten und ihre Determinanten“). Ein CochraneReview zur Frage sieht unter Einbezug von 34 Primärstudien signifikante Effekte für eine reduzierte sedentäre Arbeitszeit für die Einrichtung von Stehmöglichkeiten und für Mehrkomponenteninterventionen. Letztere Studien sind jedoch sehr heterogen [34]. Insgesamt wird dieses auch in weiteren Übersichtsarbeiten geschlussfolgert, und der Ruf nach Studien mit längerem Nachuntersuchungszeitraum, höherer Probandenzeit und Studienqualität wird einstimmig gefordert $[36,45,46]$.

Die Heterogenität wird derzeit selten über eine fehlende Theorieorientierung diskutiert $[46,47]$. Dies verwundert, da theoriebasierte Interventionen durchaus eine höhere Wirksamkeit zugeschrieben wird. Vielversprechende theoriegeleitete Zugänge, Sitzzeiten zu reduzieren, haben Gardner et al. [47] zusammengefasst. 
Sie sehen ein großes Potenzial in den Interventionsmethoden, die Selbstbeobachtung, Problemlösung und die Restrukturierung der sozialen und physischen Umwelt adressieren. Darüber hinaus würde eine stärkere Theorieorientierung auch dazu führen, den Wirkmechanismus besser zu verstehen und Interventionsmethoden und -strategien ableiten und replizieren zu können. Letztlich geht es auch um die einheitliche Benennung von Methoden und Strategien [48]. Erste Interventionsstudien wie der SMArT Work Trial schlagen einen partizipativ- und theoriegeleiten Weg ein und legen theoretisch abgeleitete Interventionsmethoden sowie Interventionsstrategien vor [49]. Die erzielten Effekte sind mit einer signifikanten Reduktion von 72 Min. pro 8 stündigen Arbeitstag gegenüber der Kontrollgruppe zudem richtungsweisend [49]. Insgesamt konnten keine Studien identifiziert werden, die die Thematik unter einer Sex/Gender-Perspektive betrachtet haben.

Im Teil II der Workshop Dokumentation [5] werden betriebliche Lösungsansätze vorgestellt. Hierfür werden neue Evaluationsergebnisse zu Änderungen der Arbeitsumgebung gezeigt. Zwei ausgewählte Beispiele geben einen Einblick in die bestehende betriebliche Praxis. Die gemeinsame Diskussion wie Interventionen am Arbeitsplatz gelingen können, gibt Hinweise wie das Thema im Rahmen der betrieblichen Prävention und Gesundheitsförderung sowie der menschengerechten Gestaltung von Arbeit weiterentwickelt werden könnte.

\section{Forschungsbedarf}

In vorliegenden Studien werden zunehmend offene Fragen bezogen auf gesundheitliche und ökonomische Folgen langer Sitzzeiten am Arbeitsplatz, Mechanismen sowie Wechselwirkungen, Gemeinsamkeiten und Unterschiede von Sitzen in Arbeit und Freizeit beschrieben. Trotz zahlreicher Studien gibt es Wissenslücken v. a. bezogen auf:

- ätiologische Studien mit hoher Qualität (v. a. mit standardisierter (objektiver) Messung der Sitzzeiten am Arbeitsplatz) und längerer Beobachtungszeit, und differenzierter Betrachtung von sedentärem Verhalten und körperlicher Aktivität

- sozial-ökologische Determinanten zum sedentären Verhalten

- theoriegeleitet, evidenzbasierte Interventionsstrategien auch unter Berücksichtigung von Diversität bzw. Gender

- weitere Evaluationen der Wirksamkeit von Maßnahmen der Arbeitsorganisation (kostenintensive vs. -extensive) und einzelnen Interventionskomponenten (z. B. soziale Unterstützung) sowie deren Verknüpfung mit gesundheitsförderlicher und menschengerechter Gestaltung von Arbeit

- laborexperimentelle Studien zur Kompensation (Dauer/ Häufigkeit/ metabolische Beanspruchung der Sitzunterbrechungen) und

- Bedarfsgerechtigkeit/Diversity von Maßnahmen zur Reduktion und Unterbrechung der Sitzzeit (in Bezug auf verschiedene Länder, Berufe in Kombination mit sozialer Ungleichheit, Altersgruppen, Geschlechter, Subgruppen mit chronischer Erkrankung).

Zukünftig sollte in den zunehmend digitalisierten Arbeitswelten untersucht werden, wie sich die wandelnden Erfordernisse in der
Arbeitswelt auf das Sitz- und Bewegungsverhalten auswirken und wie man die möglichen Chancen und Risiken mit innovativen (digitalen) Mitteln positiv beeinflussen kann.

\section{Danksagung}

Der Workshop ist Teil des Projekts Sitting@Work, das durch die Bundesanstalt für Arbeitsschutz und Arbeitsmedizin (F2399) und das Bundesministerium für Forschung und Bildung als Teil der Förderinitiative „Gesund - ein Leben lang“ (Förderkennzeichen 01GL 1721) unterstützt wird. Wir danken Herrn Prof. Dr. Jens Bucksch aus Heidelberg, Frau Gabriele Klärs aus Köln, Frau Prof. Dr. VölterMahlknecht aus Berlin und Herrn Dr. Falko Papenfuß aus Stuttgart für die konstruktiven Beiträge zur Podiumsdiskussion. Für die Organisation des Workshops danken wir Herrn Prof. Dr. Dr. Tobias Kurth und seinem Team vom Institut für Public Health an der Charité Campus Berlin-Mitte.

\section{Interessenkonflikt}

Die Autorinnen/Autoren geben an, dass kein Interessenkonflikt besteht.

Literatur

[1] Tönnies T, Röckl S, Hoyer A et al. Projected number of people with diagnosed Type 2 diabetes in Germany in 2040. Diabet Med 2019; 36 : 1217-1225 doi: 10.1111/dme.13902

[2] Reichel K, Backé E, Prigge M et al. Lange Sitzzeiten am Arbeitsplatz und kardiometabolische Gesundheit - welche Rolle spielen Genderaspekte? In: Dienstbühl I, Stadeler M, Scholle H.-C, Hrsg. Prävention von arbeitsbedingten Gesundheitsgefahren und Erkrankungen: 25. Erfurter Tage. Jena: Verlag Bussert \& Stadeler; 2019 (im Druck)

[3] Latza U. Frauengesundheit als Thema in der Arbeitsmedizin - aktuelle Perspektiven. Frauen - Arbeit - Gesundheit: Aktuelle Herausforderungen, neue Perspektiven 3. Frauengesundheitskonferenz der Bundeszentrale für gesundheitliche Aufklärung (BZgA) und des Bundesministeriums für Gesundheit (BMG), 03.09.2018, Hannover. [Online: https://www.frauengesundheitsportal.de/fileadmin/user_upload/ Frauengesundheitskonferenz_2018/Abstracts/Frauengesundheitskonferenz_2018_Abstract_Latza.pdf]

[4] Vondung C, Reichel K, Backé E et al. Sitting@Work und genEffects: Entwicklung und Anwendung von Checklisten zu „Sex/Gender“ in systematischen Reviews. Vortrag auf der 13. Jahrestagung der DGEpi am 26.09.2018 in Bremen

[5] Backé EM, Reichel K, Ellegast R et al. Workshop Gesundheitsgefährdung durch langes Sitzen am Arbeitsplatz - wissenschaftliche Perspektiven und betriebliche Lösungsansätze, Teil II Betriebliche Lösungsansätze. Gesundheitswesen. (eingereicht)

[6] Bucksch J, Schlicht W. Sitzende Lebensweise als ein gesundheitlich riskantes Verhalten. Dtsch Z Sportmed 2013; 64: 15-21

[7] Tremblay MS, Aubert S, Barnes JD et al. SBRN Terminology Consensus Project Participants. Sedentary Behavior Research Network (SBRN) - Terminology Consensus Project process and outcome. Int J Behav Nutr Phys Act 2017; 14: 75

[8] Rütten A, Pfeifer K. Nationale Empfehlungen für Bewegung und Bewegungsförderung. Friedrich-Alexander-Universität Erlangen-Nürnberg, Erlangen-Nürnberg; 2016 
[9] WHO (2010). Global Recommendations on Physical Activity for Health. Genf: World Health Organization

[10] Chau JY, Grunseit AC, Chey T et al. Daily sitting time and all-cause mortality: a meta-analysis. PLoS One 2013; 8: e80000

[11] Patterson R, McNamara E, Tainio $M$ et al. Sedentary behaviour and risk of all-cause, cardiovascular and cancer mortality, and incident type 2 diabetes: a systematic review and dose response meta-analysis. Eur J Epidemiol 2018; 33: 811-829

[12] Biswas A, Oh PIFaulkner GE et al. Sedentary time and its association with risk for disease incidence, mortality, and hospitalization in adu, Its: a systematic review and meta-analysis. Ann Intern Med 2015; 162: 123-132

[13] Ekelund U, Steene-Johannessen J, Brown W] et al. Lancet Physical Activity Series 2 Executive Committe; Lancet Sedentary Behaviour Working Group. Does physical activity attenuate, or even eliminate, the detrimental association of sitting time with mortality? A harmonised meta-analysis of data from more than 1 million men and women. Lancet 2016; 388: 1302-1310

[14] Krug S, Jordan S, Mensink GB et al. Körperliche Aktivitat: Ergebnisse der Studie zur Gesundheit Erwachsener in Deutschland (DEGS1). Bundesgesundheitsblatt Gesundheitsforschung Gesundheitsschutz 2013; 56: 765-71

[15] Booth FW, Laye M], Lees S] et al. Reduced physical activity and risk of chronic disease: the biology behind the consequences. Eur J Appl Physiol 2008; 102: 381-390

[16] Dempsey PC, Thyfault JP. Physiological responses to sedentary behaviour.In Leitzmann MF, Jochem C, Schmid S eds. Sedentary behaviour epidemiology. Cham: Springer International Publishing; 2018: 109-153

[17] Saunders TJ, Atkinson HF, Burr J et al. The acute metabolic and vascular impact of interrupting prolonged sitting: a systematic review and meta-analysis. Sports Med 2018; 48: 2347-2366

[18] Edwardson CL, Henson J, Bodicoat DH et al. Associations of reallocating sitting time into standing or stepping with glucose, insulin and insulin sensitivity: a cross-sectional analysis of adults at risk of type 2 diabetes. BMJ Open 2017; 7: e014267

[19] van Uffelen JG, Wong J, Chau JY et al. Occupational sitting and health risks: a systematic review. Am J Prev Med 2010; 39: 379-388

[20] Dragano N, Faller G, Kuhn J et al. Public Health in der Arbeitswelt. Zeitschrift Personalführung 2019; 7-8: 18-25

[21] Gesetz zur Stärkung der Gesundheitsförderung und der Prävention (Präventionsgesetz - PrävG) vom 17. Juli 2015. Bundesgesetzblatt Jahrgang 2015 Teil I Nr. 31, ausgegeben zu Bonn am 24. Juli 2015

[22] Gesetz über die Durchführung von Maßnahmen des Arbeitsschutzes zur Verbesserung der Sicherheit und des Gesundheitsschutzes der Beschäftigten bei der Arbeit (Arbeitsschutzgesetz - ArbSchG). Arbeitsschutzgesetz vom 7. August 1996 (BGBI. I S. 1246), das zuletzt durch Artikel 427 der Verordnung vom 31. August 2015 (BGBI. I S. 1474) geändert worden ist

[23] Rempel D. Do sit-stand workstations improve cardiovascular health? Occupational Medicine Forum. JOEM 2018; 60: 319-320

[24] Ng SW, Popkin BM. Time use and physical activity: a shift away from movement across the globe. Obes Rev 2012; 13: 659-680

[25] Jans MP, Proper KI, Hildebrandt VH. Sedentary behavior in Dutch workers: differences between occupations and business sectors. Am J Prev Med 2007; 33: 450-454

[26] Prince SA, Elliott CG, Scott K et al. Device-measured physical activity, sedentary behaviour and cardiometabolic health and fitness across occupational groups: A systematic review and meta-analysis. Int J Behav Nutr Phys Act 2019; 16: 30

[27] Parry S, Straker L. The contribution of office work to sedentary behaviour associated risk. BMC Public Health 2013; 13: 296
[28] Owen N, Sugiyama T, Eakin EE et al. Adults' sedentary behavior determinants and interventions. Am J Prev Med 2011; 41: 189-196

[29] Owen N, Salmon J, Koohsari MJ et al. Sedentary behaviour and health: mapping environmental and social contexts to underpin chronic disease prevention. Br J Sports Med 2014; 48: 174-177

[30] O'Donoghue G, Perchoux C, Mensah K et al. A systematic review of correlates of sedentary behaviour in adults aged $18-65$ years: a socio-ecological approach. BMC Public Health 2016; 16: 163

[31] Prince SA, Reed JL, McFetridge $C$ et al. Correlates of sedentary behaviour in adults: a systematic review. Obes Rev 2017; 18: 915-935

[32] De Cocker K, Duncan M], Short C et al. Understanding occupational sitting: Prevalence, correlates and moderating effects in Australian employees. Prev Med 2014; 67: 288-294

[33] Wallmann-Sperlich B, Bucksch J, Schneider S et al. Socio-demographic, behavioural and cognitive correlates of work-related sitting time in German men and women. BMC Public Health 2014; 14: 1259

[34] Shrestha N, Kukkonen-Harjula KT, Verbeek JH et al. Workplace interventions for reducing sitting at work. Cochrane Database Syst Rev 2018; 6: CD010912

[35] Wallmann-Sperlich B, Chau JY, Froboese I. Self-reported actual and desired proportion of sitting, standing, walking and physically demanding tasks of office employees in the workplace setting: do they fit together. BMC Research Notes 2017; 10: 504

[36] Kreis L, Backé E, Latza U. Interventionen zur Reduktion des sitzenden Verhaltens am Arbeitsplatz - ein systematischer Review. Arbeitsmed Sozialmed Umweltmed 2018; 53: 798-813

[37] Taylor WC, Suminski RR, Das BM et al. Organizational culture and implications for workplace interventions to reduce sitting time among office-based workers: a systematic review. Front Public Health 2018; 24: 6263

[38] Prince SA, Saunders T], Gresty K et al. A comparison of the effectiveness of physical activity and sedentary behaviour interventions in reducing sedentary time in adults: a systematic review and metaanalysis of controlled trials. Obes Rev 2014; 15: 905-919

[39] Martin A, Fitzsimons C, Jepson R et al. Interventions with potential to reduce sedentary time in adults: systematic review and meta-analysis. In: Br J Sports Med 2015; 49: 1056-1063

[40] Keadle SK, Conroy DE, Buman MP et al. Targeting reductions in sitting time to increase physical activity and improve health. Med Scie Sports Exerc 2017; 49: 1572-1582

[41] Bucksch J, Wallmann-Sperlich B, Kolip P. Führt Bewegungsförderung zu einer Reduzierung von sitzendem Verhalten? Präv Gesundheitsf 2015; 10: $275-280$

[42] Biddle SJ. Fit or sit? Is there a psychology of sedentary behaviour? In: Sport Exerc Psychol Rev 2011; 7: 5-10

[43] Chu AHY, Ng SHX, Tan CS et al. A systematic review and meta-analysis of workplace intervention strategies to reduce sedentary time in white-collar workers. Obes Rev 2016; 17: 467-481

[44] Peachey MM, Richardson JV Tang A et al. Environmental, behavioural and multicomponent interventions to reduce adults' sitting time: a systematic review and meta-analysis. Br J Sports Med. 2018; DOI: 10.1136/bjsports-2017-098968

[45] Backé EM, Kreis L, Latza U. Interventionen am Arbeitsplatz, die zur Veränderung des Sitzverhaltens anregen. Zbl Arbeitsmed 2019; 69: $1-10$

[46] Becker I, Wallmann-Sperlich B, Rupp R et al. Interventionen zur Reduzierung sitzenden Verhaltens am Büroarbeitsplatz - eine systematische Literaturanalyse. [Workplace Interventions to Reduce Sedentary Behavior: A Systematic Review]. Gesundheitswesen 2019; 81: 606-614 
[47] Gardner B, Smith L, Lorencatto F et al. How to reduce sitting time? A review of behaviour change strategies used in sedentary behaviour reduction interventions among adults. Health Psychol Rev 2016; 10: $89-112$

[48] Kok G, Gottlieb NH, Peters G] et al. A taxonomy of behaviour change methods: an Intervention Mapping approach. Health Psychol Rev 2016; 10: 297-312
[49] Munir F, Biddle SJH, Davies MJ et al. Stand More AT Work (SMArT Work). Using the behaviour change wheel to develop an intervention to reduce sitting time in the workplace. BMC Public Health 2018; 18: 92

[50] Buckley JP, Hedge A, Yates T et al. The sedentary office: an expert statement on the growing case for change towards better health and productivity. In: Br J Sports Med 2015; 49: 1357-1362 